\title{
Passive Wing Rotation in Flexible Flapping Wing Aerodynamics
}

\author{
Chang-kwon Kang ${ }^{1 *}$ and Wei Shyy ${ }^{2 * \dagger}$ \\ *Department of Aerospace Engineering, University of Michigan, Ann Arbor, MI, 48109 \\ ${ }^{\dagger}$ Department of Mechanical Engineering, Hong Kong University of Science and Technology, Kowloon, Hong Kong
}

\begin{abstract}
Insect wings are flexible. For rigid wings lift enhancing unsteady aerodynamics mechanisms, such as delayed stall via leading-edge vortices (LEVs), wake-capture, and rotational forces, characterize the lift generation of a hovering insect. We have uncovered a novel mechanism that fruit fly size insects can utilize to further increase the lift by adjusting its wing shape passively: A pair of a LEV and a trailing-edge vortex shed in the previous stroke induces a downward wake upstream of the wing, which acts as a wind gust and reduces the effective angle of attack. By streamlining its shape, the flexible wing is then able to mitigate the negative influence from this wing-wake interaction, resulting in a higher lift compared to its rigid counterpart. Furthermore, we show that for the flexible wings, the lift generation is the highest for the symmetric rotational mode, which is consistent with the kinematics exhibited by insects, such as fruit flies and honeybees. These results complement the existing insect flight aerodynamics and have the potential to be adapted for the development of flapping wing Micro Air Vehicles.
\end{abstract}

$\begin{array}{rlr} & \text { Nomenclature } & \\ c & =\text { chord } & {[\mathrm{m}]} \\ C_{\mathrm{L}} & =\text { lift coefficient, }-F_{2} /\left(0.5 \rho_{\mathrm{f}} U^{2}{ }_{\text {ref }} c\right) & {[1]} \\ C_{\mathrm{D}} & =\text { lateral force coefficient, } F_{1} /\left(0.5 \rho_{\mathrm{f}} U^{2}{ }_{\text {ref }} c\right) & {[1]} \\ E & =\text { Young's modulus } & {[\mathrm{Pa}]} \\ f & =\text { motion frequency } & {[1 / \mathrm{s}]} \\ f_{1} & =\text { first natural frequency of the wing } & {[1 / \mathrm{s}]} \\ f_{\mathrm{f}} & =\text { fluid force on the wing } & {[\mathrm{N} / \mathrm{m}]} \\ F_{\mathrm{i}} & =\text { fluid force acting on the wing } & {[\mathrm{N}]} \\ h & =\text { plunge motion of the wing } & {[\mathrm{m}]} \\ h_{\mathrm{a}} & =\text { plunge amplitude } & {[\mathrm{m}]} \\ h_{\mathrm{s}} & =\text { thickness of the wing } & {[\mathrm{m}]} \\ k & =\text { reduced frequency, } \pi f c / U_{\text {ref }} & {[1]} \\ p & =\text { pressure } & {[\mathrm{Pa}]} \\ Q & =\text { second invariant of the velocity gradient tensor } & {\left[1 / \mathrm{s}^{2}\right]} \\ R e & =\text { Reynolds number, } \rho_{\mathrm{f}} U_{\mathrm{ref}} c / \mu & {[1]} \\ t & =\text { time } & {[\mathrm{s}]} \\ U_{\mathrm{ref}} & =\text { reference velocity: } 2 \pi f h_{\mathrm{a}} \text { for hover } & {[\mathrm{m} / \mathrm{s}]} \\ u_{\mathrm{i}} & =\text { velocity vector } & {[\mathrm{m} / \mathrm{s}]} \\ x_{\mathrm{i}} & =\text { position vector } & {[\mathrm{m}]} \\ w & =\text { wing deflection } & {[\mathrm{m}]} \\ \alpha & =\text { angle of attack } & {[\mathrm{deg}]} \\ \alpha_{\mathrm{a}} & =\text { angular amplitude } & {[\mathrm{deg}]} \\ \alpha_{\mathrm{m}} & =\text { midstroke angle of attack } & {[\mathrm{deg}]}\end{array}$

\footnotetext{
${ }^{1}$ Postdoctoral Research Fellow, Department of Aerospace Engineering, University of Michigan, AIAA Member.

${ }^{2}$ Provost \& Chair Professor, Department of Mechanical Engineering, Hong Kong University of Science and Technology; also Adjunct Professor, Department of Aerospace Engineering, University of Michigan, AIAA Fellow.
} 


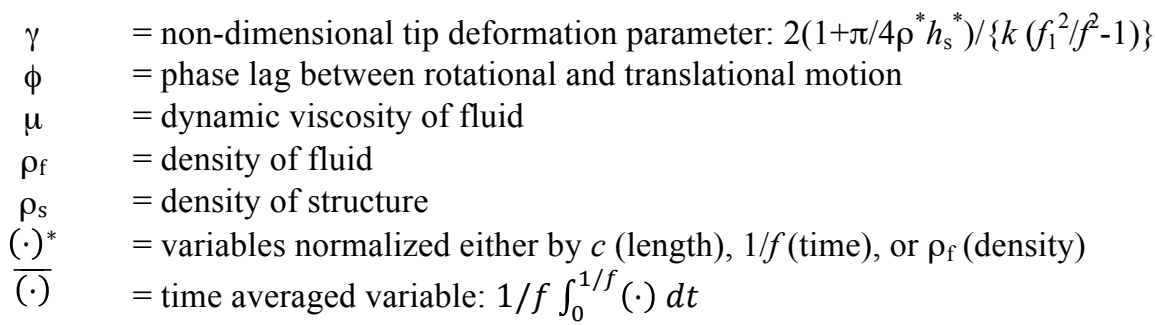

\section{Introduction}

$\mathrm{F}$ lying insects are perhaps the most banal animals in daily life, but aerodynamically they form one of the most challenging phenomena. Conventional insect flight aerodynamics has elucidated intriguing mechanisms that go beyond the steady, stationary wing theories: a novel mechanism (clap and fling ${ }^{1}$ ) based on flapping motions is found and other lift enhancing unsteady mechanisms, such as delayed stall via prolonged leading-edge vortices $(\mathrm{LEVs})^{2}$, wake-capture, and rotational forces ${ }^{3}$ are introduced to explain how the insects generate sufficient lift to stay aloft. In particular these unsteady theories predict coefficients of lift that are high enough to sustain the weights of hovering fruit flies or honey bees that operate at the Reynolds number $R e=U_{\text {ref }} c / v=100$ and 1000 , respectively,

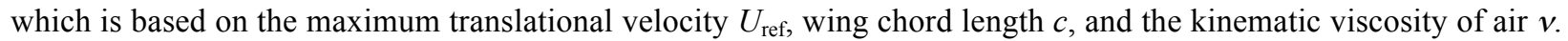
Herein plays the rotational force a fundamental role: lift is optimal when the rotation of the wing is slightly advanced relative to the translation with the midstroke angle of attack being $40^{\circ}<\alpha_{\mathrm{m}}<50^{\circ} 3,4$.

However the kinematics of a hovering fruit fly ${ }^{5}$ and a honeybee ${ }^{6}$ measured using high-speed cameras suggests that these insects rotate their wing synchronous with its translation resulting in a phase relationship called the symmetric rotation, which is also observed for beetles, dipterans, and hymenopterans ${ }^{7}$ in general. Although this phase mode is known to be near optimal ${ }^{4}$, the question has yet to be answered why the advanced rotation is not utilized in these insects.

a)
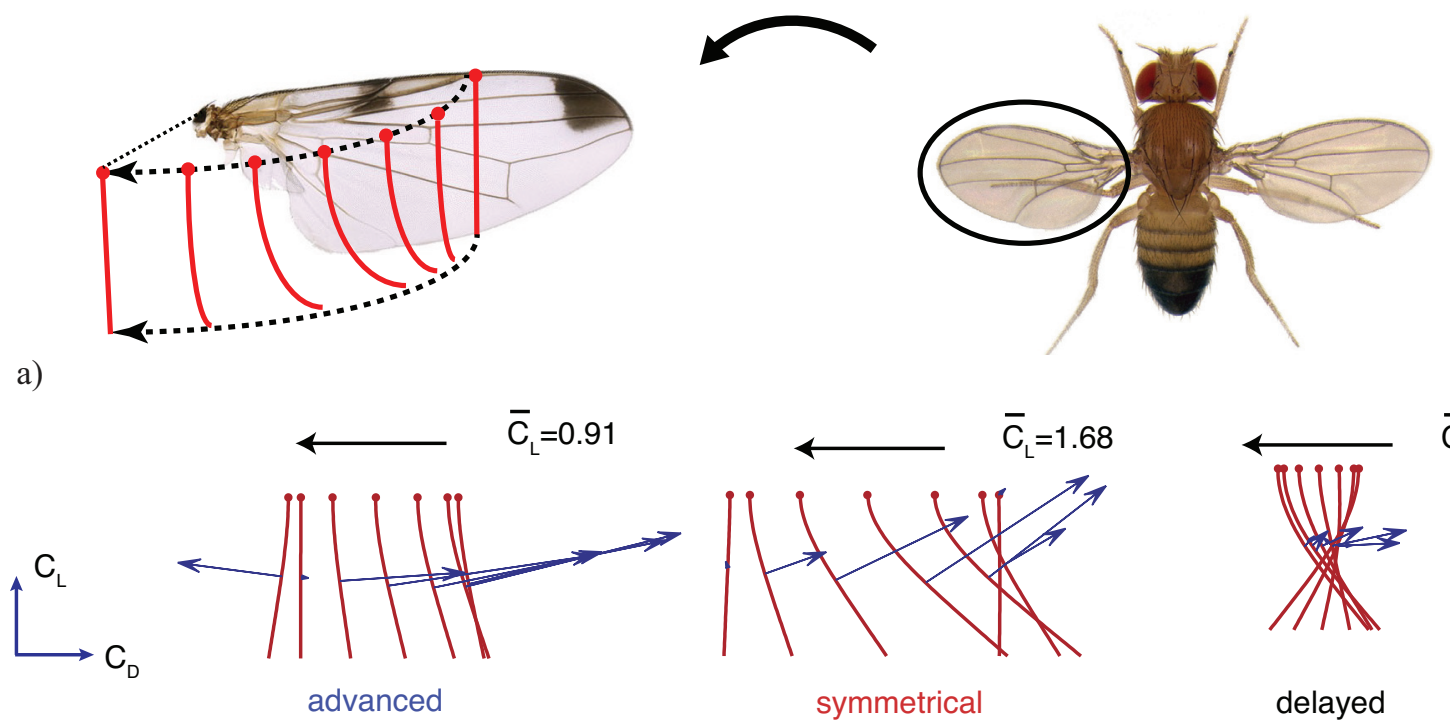

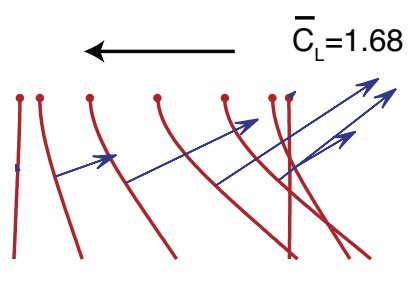

symmetrical

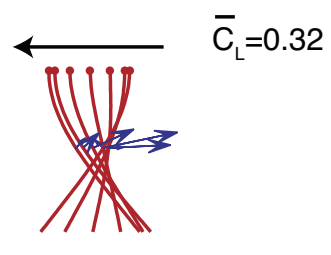

delayed

b)

Figure 1. Wing motions and aerodynamic forces generated by a flexible wing. (a) Schematic of the abstraction of the wing motion. The leading-edge is indicated by a red dot. (b) Wing shapes at 7 equally placed time instants during a backward stroke for an advanced $\left(k=0.95 ; f \mid f_{1}=0.15\right)$, symmetric $\left(k=0.6 ; f \mid f_{1}=0.25\right)$, and delayed rotation mode $\left(k=2.0 ; f / f_{1}=0.82\right)$. The resulting integrated aerodynamic forces are indicated with a blue arrow. The pictures of the Drosophila Melanogastor in (a) are with permission of Nicolas Gompel and Benjamin Prud'homme.

To address this question, we use the fact that the insect wings are flexible. The aforementioned theories assume rigid wings, while for various insect flyers are flexible: the chordwise flexural rigidity of the wing is 1 or 2 orders of 
magnitudes smaller than the spanwise flexural rigidity ${ }^{8}$. Unlike a rigid wing for which both translational and rotational are controlled actively ${ }^{3,9}$, a flexible wing can undergo passive rotation ${ }^{10}$ in dynamic balance with its inertia, aerodynamic loading, and elastic restoring force. Simplified analyses $10,11,12$ have already conjectured that the wing rotation in insect flapping may indeed be passive.

Rather than directly answering whether the wing rotation is active or passive, we consider a wing kinematics that consists of the translational motion, which is imposed on the leading-edge (LE) of the wing, and the wing rotation and camber deformations that are purely passive. It should be noted that the insects flap their wings around a pivot point (Fig. 1a), we only highlight the interplay between the chordwise flexibility and the resulting lift via wing shape deformation by approximating the wing as a homogeneous elastic twodimensional flat plate ${ }^{8}$. The three-dimensional effects, such as spanwise flow that seem to stabilize the LEVs ${ }^{13}$ and LEV-tip-vortex interaction ${ }^{14}$ could be important in general, however at $R e=\mathrm{O}\left(10^{2}\right)$ the spanwise flow is smaller than at higher Reynolds numbers ${ }^{13}$. Furthermore, the stability of the LEVs can well be approximated in twodimensions as long as the stroke-to-chord ratio is within the range that is evinced by many insects, i.e. around 4 to 5 ${ }^{15}$, which justifies the use of a two-dimensional wing ${ }^{16,17}$ and to which this study falls into.

We employ a carefully validated fully-coupled Navier-Stokes equation solver and a linear beam solver ${ }^{19,18}$ to show that by adjusting the frequency ratio $f / f_{1}$, which is the ratio between the motion frequency $f$ and the first natural frequency of the wing $f_{1}$, such passive rotation can also yield an advanced, symmetric, or delayed rotation mode (Fig. 1b), which is emphasized for the rigid wing aerodynamics with active rotation. Moreover, we highlight a mechanism that a flexible wing can utilize to outperform its rigid counterpart by enhancing the delayed stall by mitigating the wing-wake interaction via streamlining its wing shape to generate higher lift. Finally, we also show that the symmetric rotational mode yields the highest lift that is higher than of 1.6 , which is enough to make a tethered fruit fly hover ${ }^{4}$.

The current case setup is motivated by the dynamically scaled rigid wing experiments in mineral oil ${ }^{3,20}$ and we refer to our previous work ${ }^{18}$ for more details. For the insects, the wing bending is mainly due to the wing inertia as the density ratio $\rho^{*}$, the ratio between the wing density $\rho_{\mathrm{s}}$ and the fluid density $\rho_{\mathrm{f}}$, is typically of the order $\mathrm{O}\left(10^{3}\right)^{8,19}$. The mineral oil has a density that is $7 \times 10^{2}$ times greater than air, such that the acceleration-reaction force, which is often linearized by the added mass, becomes the dominant force that deforms the wing ${ }^{19}$. Still, the study of aeroelastic response of insect wings in such a low density ratio system is justified as long as the resulting aeroelasticity matches the actual motion ${ }^{20}$ as illustrated for a crane fly ${ }^{12}$ or shown by the non-dimensional relative tip deformation $\gamma$-scaling analysis ${ }^{19}$ : for the current cases the resulting lift also scales with $\gamma$ (Fig. 2).

\section{Methodology}

\section{A. Governing Equations and Kinematics}

The resulting governing equations for the incompressible fluid modeled by the unsteady two-dimensional Navier-Stokes equations with constant $\rho_{\mathrm{f}}$ and $\mu$ are 


$$
\begin{gathered}
\frac{\partial}{\partial x_{i}^{*}}\left(u_{i}^{*}\right)=0 \\
\frac{k}{\pi} \frac{\partial}{\partial t^{*}}\left(u_{i}^{*}\right)+\frac{\partial}{\partial x_{i}^{*}}\left(u_{j}^{*} u_{i}^{*}\right)=-\frac{\partial p^{*}}{\partial x_{i}^{*}}+\frac{1}{R e} \frac{\partial}{\partial x_{j}^{*}}\left\{\frac{\partial u_{i}^{*}}{\partial x_{j}^{*}}\right\}
\end{gathered}
$$

for the velocity $u_{\mathrm{i}}$, pressure $p$, position $x_{\mathrm{i}}$, time $t$, where $(\cdot)^{*}$ indicates the non-dimensional variables. The variables are non-dimensionalized with $U_{\text {ref }}$ as the velocity scale, $1 / f$ as the time scale, and $c$ as the length scale.

A horizontal sinusoidal prescribed motion is imposed on the LE of the flat plate as

$$
h^{*}\left(t^{*}\right)=h_{\mathrm{a}}^{*} \cos \left(2 \pi t^{*}\right)=\frac{1}{2 k} \cos \left(2 \pi t^{*}\right)
$$

The maximum translational velocity of LE is taken as the reference velocity $U_{\text {ref }}=2 \pi f h_{\mathrm{a}}$. The reduced frequency, which is a measure for the unsteadiness ${ }^{21}$, is $k=\pi f c / U_{\text {ref }}=1 /\left(2 h_{\mathrm{a}}{ }^{*}\right)$.

We consider a flat plate of uniform thickness oriented vertically. As the flat plate follows the imposed horizontal motion, Eq. (3), at the LE, the resulting fluid dynamic force dynamically balances with the wing inertia and the elastic bending forces, modeled locally as a linear Euler-Bernoulli beam,

$$
\frac{\partial^{2} w^{*}}{\partial t^{* 2}}+\left(\frac{k_{1}^{2} f_{1}}{2 \pi f}\right)^{2} \frac{\partial^{4} w^{*}}{\partial x_{2}^{* 4}}=\frac{f_{f}^{*}}{\rho^{*} h_{s}^{*}\left(k / \pi^{2}\right)^{2}}
$$

where $w$ is the wing deflection due to bending motion, $k_{1} \approx 1.875$ is the first spatial eigenvalue ${ }^{19}, h_{\mathrm{s}}{ }^{*}$ is the thickness ratio, $f_{f}^{*}$ the distributed transverse fluid force on the wing per unit span normalized by the inertia of the wing $\rho^{*} h_{\mathrm{s}}{ }^{*}(k / \pi)^{2}$. The aerodynamic force is normalized with $\rho_{\mathrm{f}} U_{\text {ref }}{ }^{2} c / 2$, e.g. $C_{\mathrm{L}}=-2 F_{2} /\left(\rho_{\mathrm{f}} U_{\text {ref }}{ }^{2} c\right)$ where $-F_{2}$ is the lift force and it is decomposed in the lift direction, $C_{\mathrm{L}}$, and the lateral direction, $C_{\mathrm{D}}$, see also Fig. 1.

Finally, the time-averaged values are indicated with an over-bar, which are defined as

$$
\bar{C}_{\mathrm{L}}=f \int_{m / f}^{(m+1) / f} C_{\mathrm{L}} d t,
$$

for example, for $C_{\mathrm{L}}$. The resulting forces in this study are not periodic in time and to have a representative value for the time averaged force and to avoid initial transient effects, we choose for $m=3$. In the subsequent presentation of results the non-dimensional time, $t^{*}=0.0$ represents the start of the third cycle, unless otherwise stated. For a more comprehensive treatment of the dimensional analysis and non-dimensionalization we refer to our previous work ${ }^{19}$.

\section{B. Numerical Models}

The governing equations for the fluids given by Eqs. $(1,2)$ are solved with Loci-STREAM ${ }^{22,23,24}$, which is a three-dimensional, unstructured, pressure-based finite volume solver written in a rule-based framework. It employs implicit first or second order time stepping and treats the convection terms using the second order upwind-type scheme and the pressure and viscous terms using second order schemes. The system of equations resulting from the linearized momentum equations are handled with the symmetric Gauss-Seidel solver. The pressure correction equation is solved with either the GMRES linear solver with the Jacobi preconditioner provided by PETSc ${ }^{25,26,27}$, or the BoomerAMG ${ }^{28}$ linear solver provided by hypre. The LOCI-framework is by design rule-based highly parallelizable framework for finite volume methods ${ }^{29}$. The geometric conservation law ${ }^{30}$, a necessary consideration in domains with moving boundaries, is satisfied ${ }^{31}$. The mesh deformations are realized using radial basis function (RBF) interpolations ${ }^{32}$.

An Euler-Bernoulli beam model is incorporated to solve Eq. (4) using a finite element representation. The structural damping is not considered in this study. Two degree of freedom, i.e. displacement and bending, are allowed at each node. The Newmark time integration scheme is employed. Computations done for a flexible airfoil composed of a rigid teardrop and elastic flat plate at higher Reynolds number and for various motion frequencies ${ }^{19}$ showed that a linear Euler-Bernoulli beam is sufficient for qualitative analysis of the fluid-structure interaction coupling. 
The fluid-structure interaction is based on a time-domain partitioned solution process in which the partial differential equations Eqs. $(2,4)$ governing the fluid and the structure are solved independently and spatially coupled through the interface between the fluid and the structure. An interface module is added to the fluid solver to communicate the parallelized flow solutions on the three-dimensional wetted surface to and from the serial structural solver. At each time step the fluid and the structural solvers are called one after the other until sufficient convergence on the displacements on the shared boundary surface are reached in an inner-iteration before advancing to the next time step. Full details of this algorithm and careful validation analysis against well-documented experimental results can be found in our previous work ${ }^{19}$.

\section{Case Setup}

We consider a flow with unit density initiated by a hovering two-dimensional flat plate with unit chord with $h_{\mathrm{s}}{ }^{*}$ $=0.02$ with flat edges. The fluid flow is computed with the finite volume method described in Section II.B. We refer to our previous work ${ }^{18}$ for the details of the computational setup and the temporal and spatial sensitivity tests. We focus mainly on the Reynolds number regime of a fruit fly: $R e=100$, but the qualitative trend for $R e=1000$ is similar as shown in Fig. 2. For simplicity without loss of generality we omit the presentation of $R e=1000$ cases and refer to our previous work for the Reynolds number assessment ${ }^{18}$.

The flat plate is modeled with 51 nodes equally distributed over the flat plate. The maximum translation velocity of the flat plate is such that the reference velocity $U_{\text {ref }}=2 \pi f h_{\mathrm{a}}=1$. The density ratio is $\rho^{*}=7.8$, similar to steel in water or a light material in air. The remaining non-dimensional parameters, i.e. the frequency ratio $f / f_{1}$ and the reduced frequency $k$, are varied by changing the Young's modulus $E$ and the plunge amplitude $h_{\mathrm{a}}$, respectively, to probe their influence on the resulting aerodynamics and the structural deformations. The range selection of $h_{\mathrm{a}}$ motivates from the plunge amplitudes observed from biological flyers ${ }^{9}$. It is reported in the literature that the natural flyers operate at $f \mid f_{1}<0.8^{33}$ and in this study, we adjust the Young's modulus for a similar range: $0.04<f / f_{1}<0.8$. Both ranges are extended from the previous study ${ }^{18}$ and in total 48 cases are computed for $R e=100$ and 1000 , see Table A1.

\section{Results and Discussion}

\section{A. Flexible Wing versus Rigid Wing}

Regardless of the source of the rotational motion, either actively rotated by the insect muscles, or being entirely passive due to the wing flexibility, or some combination of both, for the same wing shape and motion kinematics the resulting aerodynamic force should remain equal as the fluid dynamics are governed by the same Navier-Stokes equations. In order to assess the benefits of the passive wing rotation over its active counterpart, we extract the angles at the end and mid of the strokes from the resulting flexible wing kinematics by calculating the angle between the trailing-edge (TE) and LE of the wing. Based on these two angles a first-order harmonic ${ }^{9}$ is reconstructed for the rotational motion with the pivot point placed on the LE:

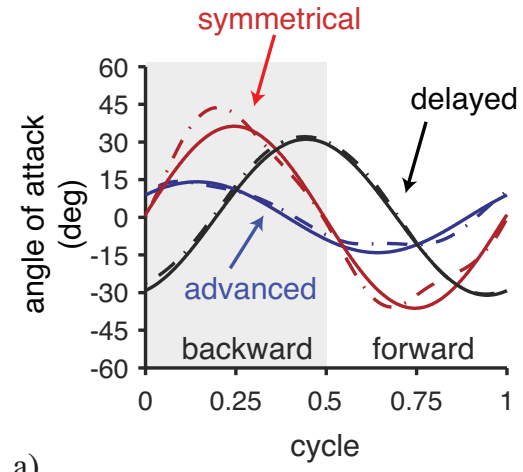

a)

b)

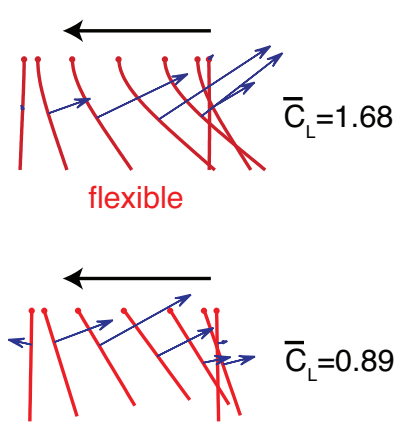

rigid

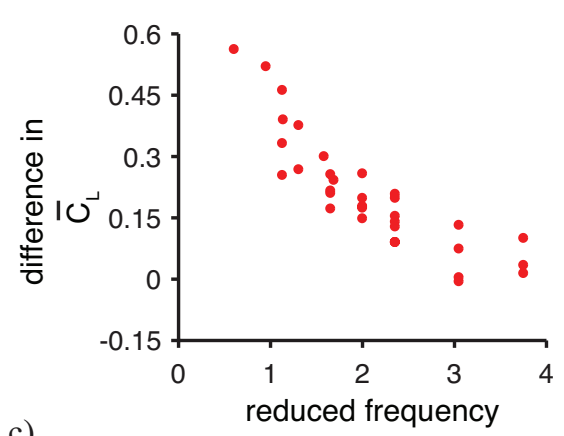

c)

Figure 3. Difference in the aeroelastic response of a hovering flexible and a rigid wing. (a) Time history of the $\alpha$ for a flexible (dash-dot) wing and its extracted active rotational motion for a rigid (solid) wing for the same cases shown in Fig. 1. (b) Instantaneous shapes of the flexible and rigid wings for the symmetric rotation case in (a). (c) Difference in the lift coefficient between the flexible and the rigid wing as a function of reduced frequency $k$. 


$$
\alpha\left(t^{*}\right)=90-\alpha_{\mathrm{a}} \cos \left(2 \pi t^{*}+\phi\right) .
$$

For the most cases the constructed rotational motion resembles the kinematics exhibited by the flexible wing closely as shown in Fig. 3a,b), however as the wing undergoes larger deformations, higher order harmonics and detailed cambering are not captured, which will result in some difference in lift.

Comparisons of the time averaged lift coefficients indicates that the most flexible wing cases yield higher lift than the rigid wings as illustrated in Fig. 3c). The largest difference is found for a symmetric rotation case with $k=$ $0.6, f / f_{1}=0.25$. Note that the cases with $f / f_{1}<$ 0.16 are omitted in this figure because for these cases the wing deformations are so small, such that the difference in lift does not follow the main trend observed. Figure 3c) further shows that this difference in lift decreases with increasing reduced frequency $k$. As $k$ increases, the influence of vortices on the force acting on the wing reduces and the acceleration-reaction force starts to dominate the force generation ${ }^{19}$. Similarly, a comparison of the unsteady lift for

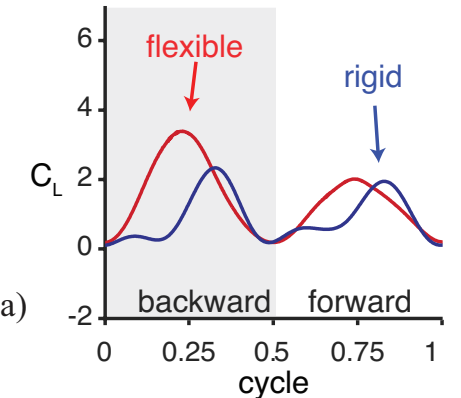

b)

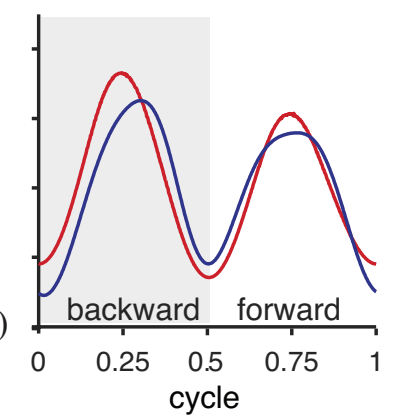

Figure 4. Time history of lift for the symmetric rotation cases (a) with a large-amplitude low-frequency motion $(k=$ $\left.0.3 ; f \mid f_{1}=0.25\right)$ and (b) with a small-amplitude highfrequency motion $\left(k=3.05 ; f / f_{1}=0.37\right)$.

the symmetric rotation cases at $k=0.6$ and $k=3.05$ illustrates that the two peaks (Fig. 4a) and larger difference in time history of lift for the rigid wing merge into one peak (Fig. 4b) for higher $k$ with smaller differences. This is because as the maximum translational velocity is kept constant, higher $k$ corresponds to greater wing acceleration, which results in greater added mass forces ${ }^{6}$.

For $k=0.6$ (Fig. 4a), the translational forces, which only depend on the instantaneous angle of attack ${ }^{3}$, dominate the lift. On the other hand, the added mass force, which is proportional to the wing acceleration and hence the reduced frequency, gains its relative contribution to the total lift at higher $k$. As such, for $k=3.05$, the added mass force term takes over ${ }^{6}$, while the mean contribution of the rotational force term, which is a coupling term between the translational velocity and the rotational velocity is neutral for the symmetric rotation mode ${ }^{34}$. This behavior is similar to the insect flight that honeybees employ short-amplitude high frequency motions (high $k$ ) and the fruit flies large-amplitude low frequency motions (low $k)^{6}$. Honeybees usually carry heavy loads due to their ecological behaviors, such that high lift generating motions are used, while the fruit flies relatively makes more use of the translational forces ${ }^{6}$.

\section{B. Wing-Wake Interaction and Streamlining.}

The lift enhancement increases for lower reduced frequencies where the translational and rotational forces are relatively greater and the nonlinear interaction between the vortices in the flow field and the wing plays a more significant role. We illustrate a mechanism in this low reduced frequency regime that leads to the lift enhancement for the flexible wing with a case that leads to the largest difference in lift (Fig. $3 \mathrm{c}: k=0.6, f / f_{1}=0.25$.). The LEV and the trailing-edge vortex (TEV) shed in the previous motion stroke form a vortex pair (Fig. 5) that induces a downward wake around the center of the stroke ${ }^{35,9}$. As soon as the wing reverses its direction at the stroke ends, wing interacts with the wake and under certain conditions ${ }^{36}$ added momentum causes the lift to increase, which is called as the wake-capture. However, when the wing passes through the downward wake, which can be regarded as wind gust, lift drops significantly for the rigid wing (Fig. 5b) ${ }^{35,9}$.

On the other hand, the flexible wing deforms its shape and adjusts itself to the surrounding flow field, such that the wing camber streamlines with the surrounding flow. Consequently, the formation of the TEVs is demoted for the flexible wing. Although the role of TEVs has not received as much interest so far as its influence on the lift generation is not directly visible for the rigid wings, for the flexible wings it has intriguing consequences. The induced downward flow is slower due to 

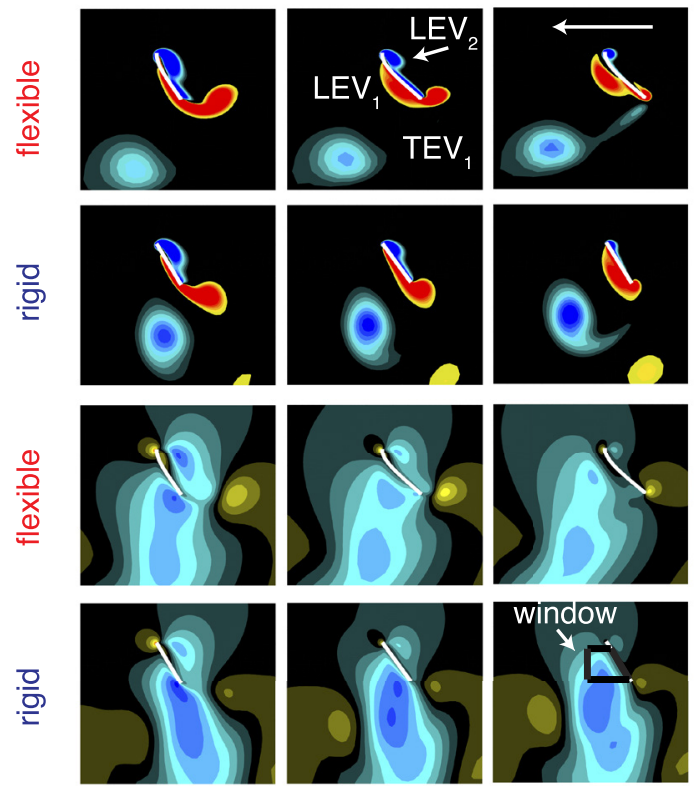

a)

5

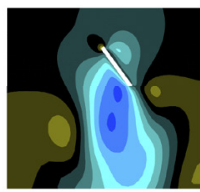

4

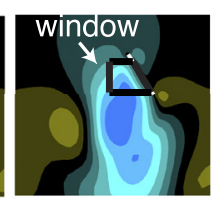

3
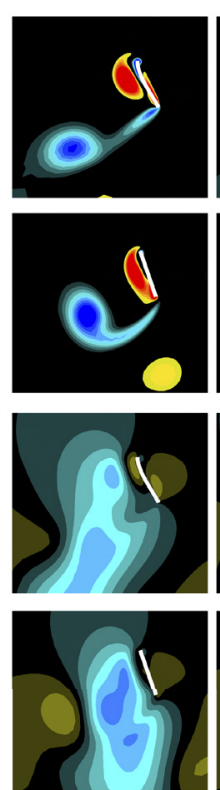

2
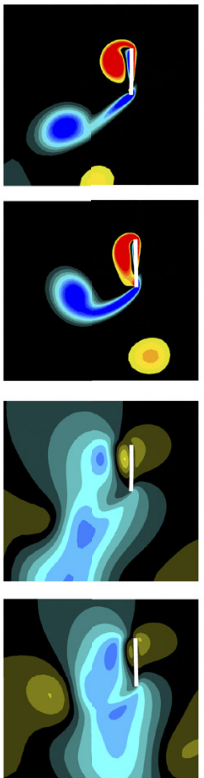

1

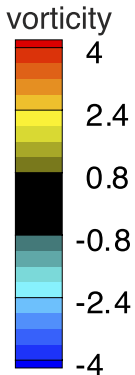

vertical velocity

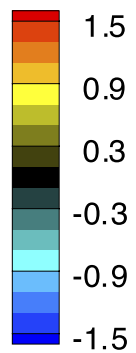

$-1.5$

c)
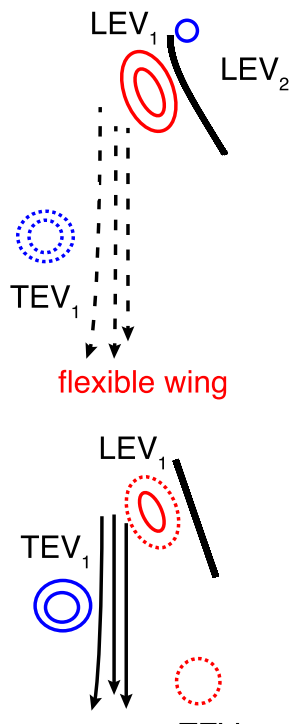

rigid wing

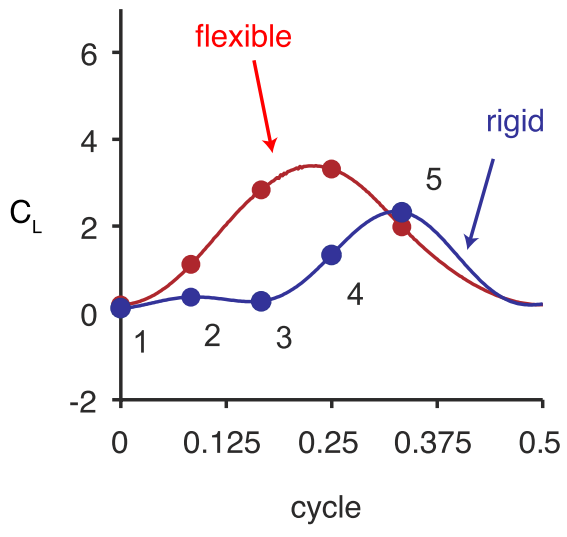

b)
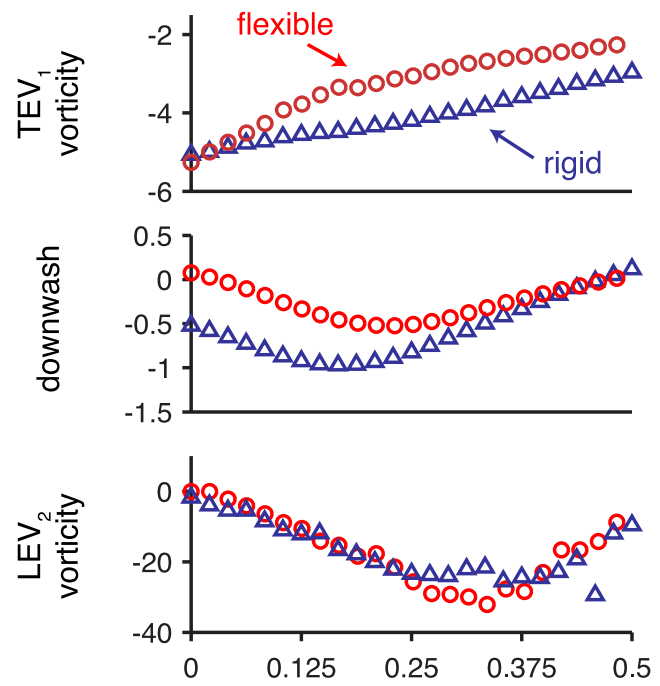

d)

cycle

Figure 5. Effects of the streamlining for the flexible wings leading to weaker TEV formation and higher lift by mitigating the strength of the downward jet, illustrated for a symmetric rotation mode $\left(k=0.6 ; f l f_{1}=0.25\right)$. (a) Vorticity and vertical velocity fields around the flexible and rigid wing. The wing thickness is exaggerated for clarity. (b) Time history of lift during the same backward stroke. The flow fields in (a) correspond to the time instants marked with the indicated numbers. (c) Schematic illustration of the wing-wake interaction. Red circles represent the positive (counter-clockwise) vorticity and blue the negative vorticity. The subscript numbers indicate the history of the stroke in which the vortices are shed: previous stroke (1) and two strokes ago (0). The subscript 2 indicates that the vortex is in formation in the current stroke. The arrows between the $\mathrm{LEV}_{1}$ and $\mathrm{TEV}_{1}$ illustrate the downwash. The velocity magnitude of the downwash illustrated by solid lines is higher than those of the dashed lines. (d) Vorticity of TEV and LEV 2 and downwash.

the weaker TEV (Fig. 5a), resulting in a higher lift for the flexible wing (Fig. 5b). Moreover, one of the effects of the wing deformation is that the orientation of the flexible wing at the LE is always normal to the translational motion of the wing, whereas the local angle of attack at the LE changes harmonically in time for the rigid wing: In the case of symmetric rotation the angle of attack at the LE is always smaller than $90^{\circ}$ (Fig. 3b), such that the formation of LEV starts earlier for the flexible wing (Fig. 5a,c). The combined result is that, while the time history of lift for the rigid 
wing depicts the traditional two peaks with the first peak accounted to the wake capture $^{3}$, the flexible wing only consists of one peak reaching its maximum at the midstroke (Fig. 4a) with the mean lift higher by 0.6 . Hence, the flexible wing can take advantage of the weaker downward wake by streamlining its TE to the flow to produce weaker TEV, which resembles the drag reduction by reconfiguration through streamlining of its flexible body ${ }^{37}$.

To quantify the lift enhancement due to this streamlining process, we first measure the vorticity at the point in the $\mathrm{TEV}_{1}$ with the highest $Q^{9}$ as illustrated in Fig. 5d). $Q$ is the second invariant of the velocity gradient tensor and higher value of $Q$

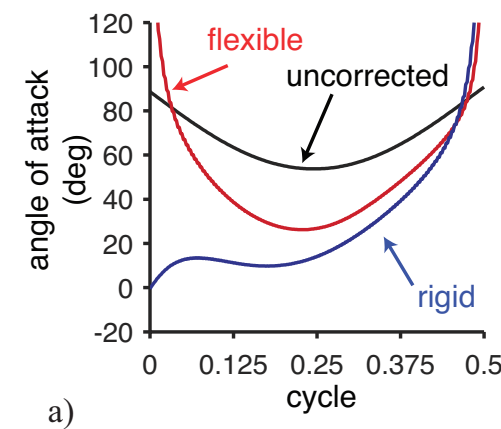

Figure 6. Effects of streamlining illustrated with quasi-steady model predictions. (a) $\alpha$ corrected for the downwash shown in Fig. 5. (b) Quasi-steady model prediction for the translational force $^{3}$ for the uncorrected (black), corrected with the downwash for the flexible (red) and the rigid (blue) wings. corresponds to greater rotation of the fluid elements. The vorticity magnitude for the rigid wing is lower for the rigid wing, which correlates to the velocity of the downwash by Biot-Savart law. Furthermore, we model the strength of the downwash by averaging the vertical velocity over a window placed upstream of the wing as shown in Fig. 5a3). This window follows the instantaneous shape of the wing shape and is fixed 0.15 chords in front of the wing chord. For various combinations of window size and positions, the qualitative trend is insensitive and the current window width and height are 0.25 and 0.75 chords, respectively. The top of the window is located at 0.25 chord location. Figure 5d) confirms the larger downwash depicted in Fig. 5a), which also leads to a stronger LEV for the flexible wing during the midstroke.

The effective angle of attack for the moving wing is a combination of the translational velocity and the downwash (Fig. 6a), which in turn affects the delayed stall of LEVs. Accordingly, the translational force components (Fig. 6b) depicts larger loss of lift for the rigid wing during around the midstroke, which the unsteady lift computed by solving the Navier-Stokes equations. On the other hand, the translational force component for the flexible wing indeed gains significantly compared to its rigid counterpart.

\section{Symmetric Rotation Optimal for Flexible Wings}

Most insects including flies, bees, and wasps employ a normal hovering, in which they flap their wings in a horizontal plane ${ }^{1,38}$. Usually, both forward and backward strokes are symmetric, generating lift in both strokes. Other hovering modes exist, e.g. the inclined hovering used by the hoverflies ${ }^{39,40}$ and dragonflies ${ }^{38}$ in which the most of the lift is generated during the downstroke; or the water-treading mode ${ }^{41}$ where the delayed stall mechanism plays the main role. For normal hovering, stroke plane deviation including the figure- 8 motions is also reported ${ }^{7}$ for biological flyers. Here, we focus on the normal hovering and its relation to passive wing rotation.

As shown in Fig. 1b), all three modes are observed for the flexible hovering flat plate even without active rotation. Moreover, the lift is optimal for the symmetric rotation (Fig. 7a), which is consistent with the wing kinematics observed for the insects. To provide a more systematic picture, we plot the phase lag $\phi$ between the rotational and translational motion, $\alpha_{\mathrm{m}}$, and lift against $f / f_{1}$ in Fig. 7b). Indeed, as $f / f_{1}$ increases the rotation first results in the advanced mode, then $\phi$ starts to decrease yielding the symmetric mode, and finally the delayed rotation modes appear. The relation between the rotational lift as a function of the phase lag $\phi$ can be seen for a sinusoidal translational motion given by Eq. (3) and the resulting rotational motion as

$$
\bar{C}_{L, \mathrm{r}} \sim \int_{0}^{1 / f} \sin (2 \pi f t) \sin (2 \pi f t+\phi) d t \sim-\cos (\phi) .
$$

Hence, the contribution of the rotational lift is positive for advanced, neutral for the symmetric, and even negative for the delayed rotations ${ }^{34}$. Moreover, albeit somewhat scattered, $\alpha_{m}$, which is indicative of the translational force component, increases with $f / f_{1}$ until the rotation begins to lag far behind the translation. The combined effect is that the resulting lift increases with $f / f_{1}$ until the rotational modes becomes symmetric, after which both the rotational lift as well as the translational lift starts to reduce for the delayed rotational modes. 
For rigid wings there are two degrees of freedom when an active rotation is imposed, e.g. angular amplitude and the phase lag. For purely passive rotations, on the other hand, the main dependent variable is the frequency ratio ${ }^{33}$, which governs both. For the cases considered in this study, the flexible wing with passive rotation is not able to achieve the advanced rotation mode with sufficiently large $\alpha_{m}$ to yield the optimal lift predicted by the rigid wing experiments ${ }^{3,4}$, because the deformations remain small for small $f / f_{1}$ to which the advanced rotation relates to (Fig. $7 \mathrm{~b}$ ). Rather, because of the competing trend of increasing $\alpha_{\mathrm{m}}$ for $f / f_{1}<0.5$ and decreasing $\phi$ with $f / f_{1}$, the optimal lift is reached for the symmetric rotation with $0.25<f l f_{1}<0.4$, where the TE motion is in phase with the translational motion: the higher angle of attack due to larger deformations coincides with the midstroke at which the translational velocity is maximal (Fig. 7c). The resulting lift coefficient is higher than 1.6, which is sufficient to sustain the weight of a tethered fruit fly ${ }^{4}$.

These results suggest that at these Reynolds numbers when the only rotational mechanism is due to passive wing rotation, then the optimal aerodynamic performance correlates to symmetric rotation motion and the phasing can be controlled by adjusting the frequency ratio. Compared to the rigid wing cases ${ }^{3}$ the achieved lift is higher, even though active rotation is not required. This excursion to the flexible flapping wings evinces promising results that complement the existing insect flight aerodynamics and that can be readily adapted for development of flapping wing Micro Air Vehicles (MAVs), where one of the main challenges of designing flapping MAVs is the weight penalty from implementing the two motion actuators, one for translation (or flapping) and one for pitching.

a)
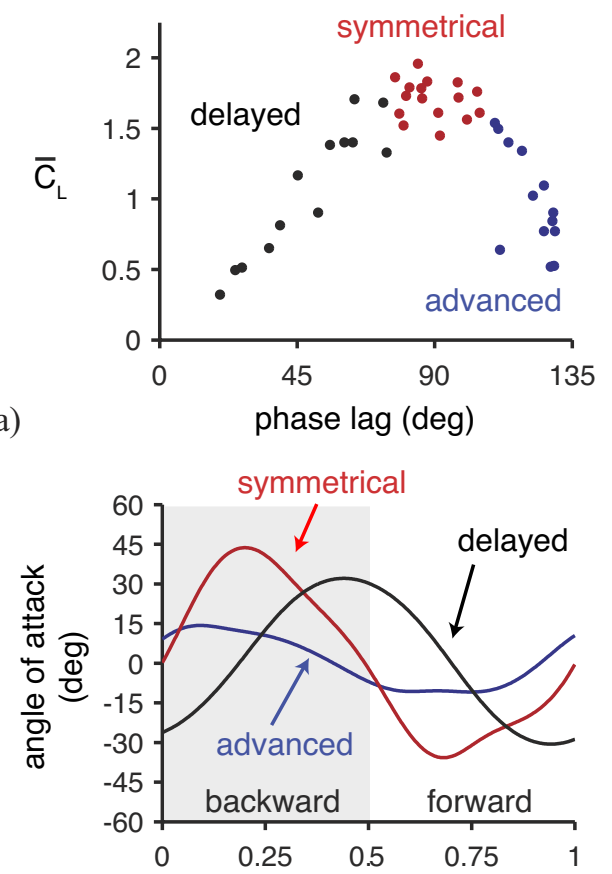

c)

cycle

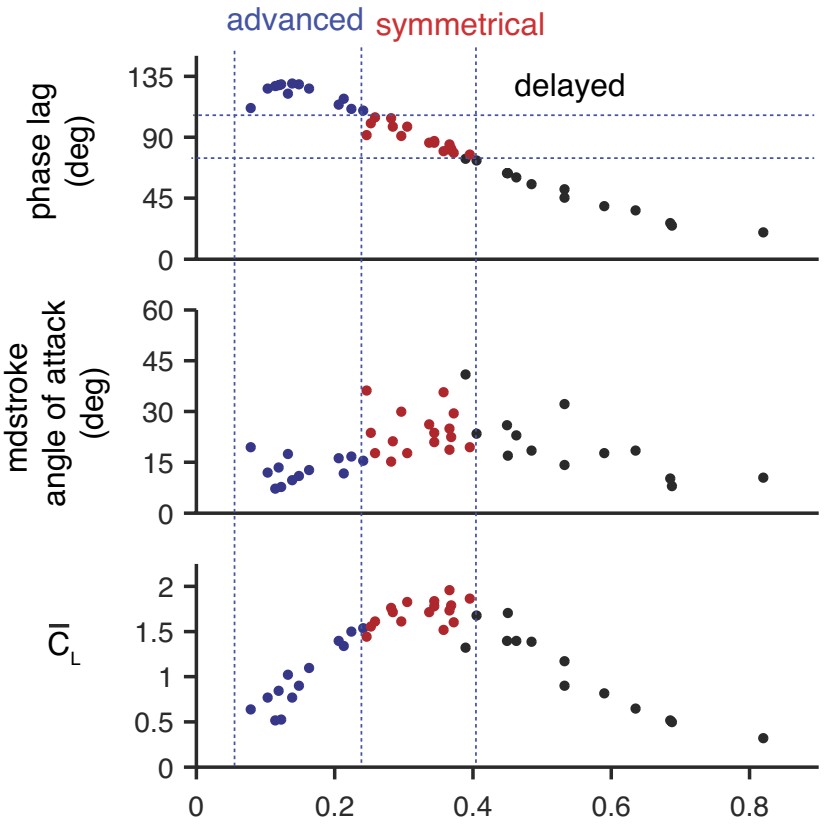

b)

frequency ratio

Figure 7. Aeroelastic response of a hovering flexible. (a) Lift coefficient as a function of $\phi$. (b) $\phi$, $\alpha_{\mathrm{m}}$, and lift coefficient as a function of $f / f_{1}$. (c) Time history of angle of attack due to passive rotation.

\section{Conclusion}

We use a fully coupled Navier-Stokes equation solver and Euler-Bernoulli beam solver to uncover new unsteady aerodynamics mechanisms applicable for an insect operating at $R e=100$ to 1000 . For a flexible wing, the difference in the detailed camber due to the wing shape deformation leads a higher lift compared to its rigid counterpart. Although for both types of wing the same translational motion is imposed at the LE of the wing, the rotational motion is a result of the dynamic balance between the wing inertia, fluid dynamic force, and the elastic restoring force for the flexible wing, while for the rigid wing the rotation is prescribed.

The streamlining of the wing shape for the flexible wing results in a TEV formation that is weaker than for the rigid wing. Consequently, the induced downwash in the upstream of the motion is mitigated for the flexible wing leading to a reduced wing-wake interaction in the upcoming stroke. The downwash acts as a wind gust that lowers 
the effective angle of attack. As a result, the LEV formation is stronger for the flexible wing and also the lift is higher.

The enhanced performance for the flexible wing decreases with reduced frequency. At higher reduced frequencies, e.g. low-amplitude high frequency motions employed by honeybees, the acceleration of the wing increases and the acceleration-reaction force dominates the aerodynamic force generation. Hence, when the wing kinematics is similar, the resulting force magnitudes are similar. However, at lower reduced frequencies, e.g. largeamplitude low frequency motions such as for the fruit flies, the influence of the vortex dynamics and wing-wake interaction gains relatively more importance and the mechanisms described above result in greater lift generation for the flexible wing.

Finally, we show that for the cases considered the symmetric rotation mode yields the highest lift for the flexible wings compared the motions that result in delayed or advanced rotations. This finding is consistent with the kinematics exhibited by fruit flies and honeybees. The achieved lift is sufficiently high to sustain the weight of a fruit fly.

\section{Acknowledgments}

This work was supported by the Air Force Office of Scientific Research's Multidisciplinary University Research Initiative grant.

\section{Appendix}

\section{A. Case Descriptions}

Table A1. List of data points.

\begin{tabular}{ccc|ccc|ccc}
\hline \hline Case & $\boldsymbol{k}$ & $\boldsymbol{f} \mid \boldsymbol{f}_{\mathbf{1}}$ & Case & $\boldsymbol{k}$ & $\boldsymbol{f} \boldsymbol{|} \boldsymbol{f}_{\mathbf{1}}$ & Case & $\boldsymbol{k}$ & $\boldsymbol{f}_{\boldsymbol{f} \mathbf{1}}$ \\
\hline 1 & 0.25 & 0.08 & 17 & 0.95 & 0.16 & 33 & 0.95 & 0.14 \\
2 & 2.00 & 0.64 & 18 & 1.30 & 0.22 & 34 & 1.65 & 0.24 \\
3 & 0.25 & 0.05 & 19 & 1.65 & 0.28 & 35 & 2.35 & 0.34 \\
4 & 2.00 & 0.37 & 20 & 2.00 & 0.34 & 36 & 0.95 & 0.12 \\
5 & 1.13 & 0.36 & 21 & 2.35 & 0.59 & 37 & 1.65 & 0.21 \\
6 & 1.13 & 0.21 & 22 & 2.35 & 0.46 & 38 & 2.35 & 0.30 \\
7 & 0.25 & 0.06 & 23 & 2.35 & 0.40 & 39 & 3.05 & 0.40 \\
8 & 2.00 & 0.45 & 24 & 0.60 & 0.25 & 40 & 3.05 & 0.48 \\
9 & 1.13 & 0.25 & 25 & 0.95 & 0.39 & 41 & 3.05 & 0.69 \\
10 & 1.68 & 0.34 & 26 & 1.30 & 0.53 & 42 & 0.95 & 0.11 \\
11 & 0.48 & 0.13 & 27 & 2.00 & 0.82 & 43 & 2.35 & 0.28 \\
12 & 1.14 & 0.30 & 28 & 0.25 & 0.04 & 44 & 3.75 & 0.45 \\
13 & 0.63 & 0.12 & 29 & 0.95 & 0.15 & 45 & 3.75 & 0.53 \\
14 & 1.58 & 0.37 & 30 & 1.65 & 0.26 & 46 & 3.75 & 0.69 \\
15 & 0.25 & 0.04 & 31 & 2.35 & 0.37 & 47 & 3.05 & 0.37 \\
16 & 0.60 & 0.10 & 32 & 0.25 & 0.04 & 48 & 0.25 & 0.03 \\
\hline \hline
\end{tabular}

\section{References}

${ }^{1}$ Weis-Fogh, T., "Quick Estimates of Flight Fitness in Hovering Animals, including Novel Mechanism for Lift Production," Journal of Experimental Biology, Vol. 59, pp. 169 - 230, 1973.

${ }^{2}$ Ellington, C. P., van der Berg, C., Willmott, A. P., and Thomas, A. L.R., "Leading-edge Vortices in Insect Flight," Nature, Vol. 384, pp. 626 - 630, 1996.

${ }^{3}$ Dickinson, M. H., Lehmann, F.-O., and Sane, S. P., "Wing Rotation and the Aerodynamic Basis of Insect Flight," Science, Vol. 284, pp. 1954 - 1960, 1999.

${ }^{4}$ Sane, S. P. and Dickinson, M. H., "The Control of Flight Force by a Flapping Wing: Lift and Drag," Journal of Experimental Biology, Vol. 204, pp. 2607 - 2626, 2001. 
${ }^{5}$ Fry, S. N., Sayaman, R., and Dickinson, M. H., "The Aerodynamics of Hovering Flight in Drosophila," J. Exp. Biol., Vol. 208, pp. 2303-2318, 2005.

${ }^{6}$ Altshuler, D. L., Dickson, W. B., Vance, H. T., Roberts, S. P., and Dickinson, M. H., "Short-ampllitude Highfrequency Wing Strokes determine the Aerodynamics of Honeybee Flight," Proceedings of National Academy of Sciences U.S.A., Vol. 102, pp. 18213-18218, 2005.

${ }^{7}$ Lehmann, F.-O., Gorb, S., Nasir, N., and Schützner, P., "Elastic Deformation and Energy Loss of Flapping Fly Wings," Journal of Experimental Biology, Vol. 214, pp. 2949 - 2961, 2011.

${ }^{8}$ Combes, S. A. and Daniel, T. J., "Flexural Stiffness in Insect Wings I. Scaling and the Influence of Wing Venetion," Journal of Experimental Biology, Vol. 206, pp. 2979-2987, 2003.

${ }^{9}$ Trizila, P., Kang, C.-K., Aono, H., Shyy, W., and Visbal, M., "Low-Reynolds Number Aerodynamics of a Flapping Rigid Flat Plate," AIAA Journal, Vol. 49, pp. 806-823, 2011.

${ }^{10}$ Ennos, A. R., "The Inertial Cause Of Wing Rotation in Diptera," Journal of Experimental Biology, Vol. 140, pp. $161-169,1988$.

${ }^{11}$ Bergou, A. J., Xu, S., and Wang, Z. J., "Passive Wing Pitch Reversal in Insect Flight," Journal of Fluid Mechanics, Vol. 591, pp. 321-338, 2007.

${ }^{12}$ Ishihara, D., Yamashita, Y., Horie, T., Yoshida, S., and Niho, T., "Passive Maintenance of High Angle of Attack and its Lift Generation during Flapping Translation in Crane Fly Wing," Journal of Experimental Biology, Vol. 212, pp. 3882-3891, 2009.

${ }^{13}$ Birch, J. M. and Dickinson, M. H., "Spanwise Flow and the Attachment of the Leading-edge Vortex on Insect Wings," Nature, Vol. 412, pp. 729-733, 2001.

${ }^{14}$ Shyy, W., Trizila, P., Kang, C.-K., and Aono, H., "Can Tip Vortices enhance Lift of a Flapping Wing?," AIAA Journal, Vol. 47, pp. 289-293, 2009.

${ }^{15}$ Wang, Z. J., "Dissecting Insect Flight," Annnual Review of Fluid Mechanics, Vol. 37, pp. 183 - 210, 2005.

${ }^{16}$ Wang, Z. J., Birch, J. M., and Dickinson, M. H., "Unsteady Forces and Flows in low Reynolds Number Hovering Flight: Two-dimensional Computations vs Robotic Wing Experiments," Journal of Experimental Biology, Vol. 207, pp. 449 - 460, 2004.

${ }^{17}$ Alben, S. and Shelley, M., "Coherent Locomotion as an Attracting State for a Free Flapping Body," Proceedings of National Academy of Sciences U.S.A., Vol. 102, pp. 11163-11166, 2005.

${ }^{18}$ Kang, C.-K. and Shyy, W., "Effects of Flexibility on the Aerodynamics of a Hovering Flexible Airfoil at Reynolds Number of 100 to 1000 ," in 50th AIAA Aerospace Sciences Meeting including the New Horizons Forum and Aerospace Exposition, Nashville, Tennessee, Jan. 9-12, 2012, 2012, pp. AIAA-2012-1206.

${ }^{19}$ Kang, C., Aono, H., Cesnik, C. E.S., and Shyy, W., "Effects of Flexibility on the Aerodynamic Performance of Flapping Wings," Journal of Fluid Mechanics, Vol. 689, pp. 32 - 74, 2011.

${ }^{20}$ Zhao, L., Huang, Q., Deng, X., and Sane, S., "Aerodynamic Effects of Flexibility in Flapping Wings," Journal of Royal Society Interface, Vol. 7, pp. 485-497, 2010.

${ }^{21}$ Triantafyllou, M. S., Triantafyllou, G. S., and Yue, D. K.P., "Hydrodynamics of Fishlike Swimming," Annual Review of Fluid Mechanics, Vol. 32, pp. 33 - 53, 2000.

${ }^{22}$ Wright, J. A. and Smith, R. W., "An Edge-based Method for the Incompressible Navier-Stokes Equations on Polygonal Meshes," Journal of Computational Physics, Vol. 169, pp. 24 - 43, 2001.

${ }^{23}$ Smith, R. W. and Wright, J. A., "An Implicit Edge-based ALE Method for the Incompressible Navier-Stokes Equations," International Journal of Numerical Methods in Fluids, Vol. 43, pp. 253 - 279, 2003.

${ }^{24}$ Kamakoti, R., Thakur, S., Wright, J., and Shyy, W., "Validation of a New Parallel All-Speed Cfd Code in a Rulebased Framework for Multidisciplinary Applications," in 36th AIAA Fluid Dynamics Conference and Exhibit, San Francisco, California, June 5-8, 2006, AIAA-2006-3063.

${ }^{25}$ Balay, S., Gropp, W. D., McInnes, L. C., and Smith, B. F., "Efficient Management of Parallelism in Object Oriented Numerical Software Libraries," in Modern Software Tools in Scientific Computing, 1997, pp. 163-202.

${ }^{26}$ Balay, S. et al., "PETSc Users Manual," Argonne National Laboratory, 2010.

${ }^{27}$ S. Balay, J. Brown, K. Buschelman, W. D. Gropp, D. Kaushik, M. G. Knepley, L. C. McInnes, B. F. Smith, and H. Zhang. (2011) PETSc Web page. [Online]. http://www.mcs.anl.gov/petsc

${ }^{28}$ Falgout, R. and Yang, U., "hypre: A Library of High Performance Preconditioners," in Computational Science ICCS 2002, Sloot, P., Hoekstra, A., Tan, C., and Dongarra, J.: Springer Berlin / Heidelberg, 2002, pp. 632-641.

${ }^{29}$ Luke, E. A. and George, T., "Loci: a Rule-based Framework for Parallel Multi-disciplinary Simulation Synthesis," Journal of Functional Programming, Vol. 15, Nr. 03, pp. 477 - 502, 2005. 
${ }^{30}$ Thomas, P.D. and Lombard, C. K., "Geometric Conservation Law and its Application to Flow Computations on Moving Grids," AIAA Journal, Vol. 17, Nr. 10, pp. 1030 - 1037, 1979.

${ }^{31}$ Kamakoti, R. and Shyy, W., "Evaluation of Geometric Conservation Law using Pressure-based Fluid Solver and Moving Grid Technique," International Journal of Heat and Fluid Flow, Vol. 14, Nr. 7, pp. 851 - 865, 2004.

${ }^{32}$ Boer, A. d., van, M. S., and Bijl, H., "Mesh Deformation Based on Radial Basis Function Interpolation," Computers \& Structures, Vol. 85, Nr. 11-14, pp. 784 - 795, 2007.

${ }^{33}$ Ramananarivo, S., Godoy-Diana, R., and Thiria, B., "Rather than Resonance, Flapping Wing Flyers May Play on Aerodynamics to Improve Performance," Proceedings of the National Academy of Sciences, Vol. 108, Nr. 15, pp. 5964 - 5969, 2011.

${ }^{34}$ Sane, S. and Dickinson, M. H., "The Aerodynamic Effects of Wing Rotation and a Revised Quasi-steady Model of Flapping Flight," Journal of Experimental Biology, Vol. 205, pp. 1087-1096, 2002.

${ }^{35}$ Birch, J. M. and Dickinson, M. H., "The Influence of Wing-wake Interactions on the Productions of Aerodynamic Forces in Flapping Flight," Journal of Experimental Biology, Vol. 206, pp. 2257 - 2272, 2003.

${ }^{36}$ Lua, K. B., Lim, T. T., and Yeo, K. S., "Effect of Wing-wake Interaction on Aerodynamic Force Generation on a 2D Flapping Wing," Experiments in Fluids, Vol. 51, Nr. 1, pp. 177-195, 2011.

${ }^{37}$ Alben, S., Shelley, M., and Zhang, J., "Drag Reduction through Self-similar Bending of a Flexible Body," Nature, Vol. 420, pp. 479-481, 2002.

${ }^{38}$ Wang, Z. J., "The Role of Drag in Insect Hovering," Journal of Experimental Biology, Vol. 207, pp. 4147 - 4155 , 2004.

${ }^{39} \mathrm{Du}, \mathrm{G}$. and Sun, M., "Effects of Wing Deformation on Aerodynamic Forces in Hovering Hoverflies," Journal of Experimental Biology, Vol. 213, pp. 2273 - 2283, 2010.

${ }^{40}$ Mou, X. L., Liu, Y. P., and Sun, M., "Wing Motion Measurement and Aerodynamics of Hovering True Hoverflies," Journal of Experimental Biology, Vol. 214, pp. 2832 - 2844, 2011.

${ }^{41}$ Freymuth, P., "Thrust Generation by an Airfoil in Hover Modes," Experiments in Fluids, Vol. 9, pp. 17 - 24, 1990. 